\title{
Examination of the Impact of Condensed Biofeedback Training on Acute Stress Responses
}

\author{
Meredith Carroll11, Brent Winslow ${ }^{2}$ \\ ${ }^{1}$ College of Aeronautics, Florida Institute of Technology, Melbourne, FL, USA \\ ${ }^{2}$ Design Interactive, Inc., Orlando, FL, USA \\ Email: mcarroll@fit.edu
}

How to cite this paper: Carroll, M. and Winslow, B. (2017) Examination of the Impact of Condensed Biofeedback Training on Acute Stress Responses. Journal of Behavioral and Brain Science, 7, 287-303. https://doi.org/10.4236/jbbs.2017.77021

Received: May 27, 2017

Accepted: July 9, 2017

Published: July 13, 2017

Copyright (c) 2017 by authors and Scientific Research Publishing Inc. This work is licensed under the Creative Commons Attribution International License (CC BY 4.0).

http://creativecommons.org/licenses/by/4.0/ (c) (i) Open Access

\begin{abstract}
The objective of this study was to measure the effects of a condensed 90 minute Biofeedback Training (BFT) method on stress response and decision making performance under stress. Forty one novice male participants received either BFT training, which incorporated diaphragmatic breathing with Stress Inoculation Training (SIT), or a control training task. Participants completed pre- and post-training assessments which incorporated a socio evaluative stress induction method followed immediately by performance of a simulation-based decision making under stress scenario. Stress was assessed using real-time physiological measures of the Autonomic Nervous System (ANS) response and cortisol measures of the Hypothalamic-Pituitary-Adrenal (HPA) axis stress response. Perceived stress was measured using the state portion of the State Trait Anxiety Inventory and decision making performance was assessed during scenario performance. Results showed that participants in the BFT condition experienced a significant reduction in cortisol from pre-training to post-training, while the control group did not. However, BFT participants did not experience statistically significant reductions in ANS stress response or in perceived stress compared to the control group. Participants in the biofeedback group experienced greater improvements in performance from pre-training to post-training compared to the control group; however, these results only approached statistical significance $(p=0.09)$. These results suggest that the condensed BFT method has the potential to impart the knowledge and skills necessary to implement the biofeedback-based coping mechanisms; however, it may require additional practice time to allow the technique to be utilized more effectively.
\end{abstract}

\section{Keywords}

Physiological Stress, Biofeedback, Stress Training, Cortisol 


\section{Introduction}

Stress is a central aspect of human life. While exposure to moderate, acute stress is associated with improved performance, long-term severe stress can reduce fine motor performance [1], attention [2], and cognitive function [3] due to biological and neural mechanisms. Unmitigated stress is associated with a number of disease states including cardiovascular disease, depression, and cancer [4], and with a significant reduction in productivity [5]. Extended exposure to stress or exposure to severe traumatic stressors are associated with an increased likelihood of developing clinical stress disorders such as major depressive disorder (MDD), post-traumatic stress disorder (PTSD), or suicide ideation and attempt [6].

One group that may be at increased risk for stress-related illness is active duty military. Previous research has indicated that survival training and battlefield stress impairs working memory, reaction time, and visual-spatial capacity which can lead to operational or battlefield errors [1] [7]. Combat stress has also been shown to affect midbrain activity and attention, including reduced functional connectivity between the prefrontal cortex and midbrain, which persisted at an 18 month follow-up [3]. Surging military rates of PTSD [8] and suicide in the wake of the US military operations in the Middle East [9], illustrate the need for training to prepare individuals to recognize and cope with severe stress both during performance and in the aftermath.

There are a range of training methods that have shown to be effective in improving an individual's ability to cope with stress. Training programs such as mindfulness-based training [10] [11] which incorporate aspects of meditation, breathing, and yoga exercises, have shown positive impacts on the ability of individuals to cope with and recover from stress, including stressors faced in military operations [12]. Cognitive behavioral therapy (CBT), a method based on the belief that dysfunctional behaviors are often cognitively mediated, and which therefore targets the dysfunctional thinking, has been shown effective in reducing PTSD symptoms and depression and anxiety [13] [14]. An emerging method which has been shown effective in mitigating stress effects across multiple stressor and implementation methods is biofeedback training.

\section{Biofeedback Training}

Biofeedback is a process by which individuals learn to identify and alter autonomic nervous system (ANS) functions such as cardiovascular activity, muscle tone, or sweat activity by monitoring physiological responses to stress [15]. Biofeedback Training (BFT) techniques typically encompass three stages wherein a trainee 1) acquires awareness of maladaptive physiological responses, 2) learns to control the response utilizing techniques such as deep breathing and passive muscle relaxation and 3) learns to transfer this control to everyday life [15]. BFT has been implemented with a wide range of physiological monitoring equipment as well as via manual self-monitoring with the goal of enabling participants to integrate the methods into their everyday life without the aid of monitoring technology [15]. 
has been shown effective in reducing stress associated with a range of different medical conditions including anxiety, chronic pain, epilepsy and hypertension [16], as well as stress associated with everyday hassles [17], military combat [18], and sports competitions [19]. These successes have been demonstrated across a broad range of implementation techniques, including in conjunction with physical therapy [20] and psychotherapy [21]. BFT is often implemented in the context of stress exposure strategies such as Stress Inoculation Training (SIT) [22], Stress Exposure Training (SET) [23] and Stress Management Training (SMT) [24] [25], techniques which incorporate a similar three phase approach, that involves: 1) an education phase to help the trainee better understand the nature of stress and stress effects, 2) a skill acquisition and rehearsal phase to facilitate development and practice a repertoire of coping skills and 3) an application phase in which coping skills are applied in conditions that increasingly approximate the transfer environment. Each of these techniques have been shown to be effective in increasing a trainee's ability to cope with stress both in isolation [22] [24] [26] [27] as well as when combined with BFT [24] [28]. For example, one group recently conducted a study in which biofeedback was used in conjunction with the stress management skill of diaphragmatic breathing during a horror first person shooter (FPS) videogame [24]. Prior to this measure, participants assigned to the SMT condition were able to practice the breathing technique while playing a videogame. Those involved in this condition had significantly lower salivary cortisol (a key physiological indicator of hypothalamic-pituitaryadrenal [HPA] axis stress response) both immediately before and after the simulation when compared to a control group. The participants were adequately trained in SMT; all had previously received SMT prior to the experiment, and the experimental process provided participants with three separate 30-minute training sessions as well as 15-minute refresher briefings over the course of three days.

One challenge with this method is that general guidelines for BFT state that it is necessary for individuals to take an active role in practicing in order to develop the skill [16]. Although there are discrepancies between the length of time needed for practice, many successful BFT studies, which have found significant reductions in physiological and perceived stress response, incorporate multiple training sessions in order to ensure that participants properly acquire the new technique they practiced [21] [24] [29] [30]. Further, much of the previous BFT research has utilized significant training time (e.g., multiple weeks of training) to produce significant quantitative and qualitative outcomes [17] [31] [19] [32].

Often, in domains such as the military and given the current pace of our culture, training that requires participation over weeks or even multiple days may prove infeasible or enough of a barrier that the training will not be pursued. If a BFT method could be condensed into a single short session, individuals would be more likely to participate and organizations such as the military would be more likely to implement it. However, there are only a limited number of studies exploring whether or not stress coping skills can be effectively trained utilizing 
such a condensed BFT method and if such a method can facilitate the same reductions in physiological stress response and perceived stress. One group examined the impact of a 90 minute BFT/SIT method on participants who rated high in test anxiety and, when compared to no training, reported significant decreases in test anxiety (via the Test Anxiety Index) and perceived stress (via the Autonomic Perception Questionnaire and the State-Trait Anxiety Inventory (STAI) ) [33]. Another group examined the impact of BFT on military participants during pre-deployment training and found that a 20 minute BFT session resulted in significant decreases in ANS response, as measured by HRV, during assessment in a multimedia combat stress environment. These studies suggest that a condensed BFT may provide adequate training to facilitate participants' successful learning and implementation of the biofeedback technique to reduce subsequent stress response [18].

\section{Research Study Objectives}

The objective of this experimental study was to measure the effects of a condensed 90 minute BFT training method, which incorporates SIT and diaphragmatic breathing, on stress response and decision making performance under stress. To achieve this, participants received a socio evaluative stress induction technique immediately followed by performance of a simulated decision making under stress task, both before and after receiving training per their treatment condition (either BFT or a control training task). Physiological stress response (including indicators of both ANS and HPA stress response) and performance were monitored during the pre- and post-training phases and changes across the training sessions were compared between groups to determine the effectiveness of the condensed BFT method. It was hypothesized that the BFT condition would demonstrate significant decreases in stress response and significant increases in performance from pre-training to post-training compared to the control condition.

\section{Methods}

\subsection{Participants}

All methods involving participants were approved by an independent Institutional Review Board (Copernicus Group, Durham, NC). All participants were recruited from ads distributed at local universities and were prescreened in a telephone interview to ensure that they met minimum requirements including age (18 - 35), normal visual acuity, and no medical conditions such as endocrine disorders. Forty one novice male participants [average age $21.0 \pm 2.2$ (SD) years] completed and received payment of $\$ 100$ USD for participation in the study. Further, all participants scored within the normal range of the Connor-Davidson Resilience Scale (CD-RISC), indicating normal stress coping abilities. Exclusion criteria were chosen to approximate US Marine small unit leaders.

\subsection{Experimental Design}

The experimental design was a between subjects repeated measures design with a 
between subjects independent variable of training type (BFT vs. control) and a within subjects independent variable of trial (pre-training vs. post-training). There were 21 participants in the BFT group and 20 participants in the control group.

\subsection{Experimental Procedure}

The experimental procedure is overviewed in Figure 1 below. Participants arrived between 8:00 and 8:30 AM, provided written informed consent, completed a demographics questionnaire, and the state portion of the STAI [34], previously shown to have a high test-retest reliability with situational stress [35]. Wireless physiological sensors were then placed on the participants, followed by a fiveminute recording of baseline physiological activity while participants remained seated. Participants were instructed prior to arrival to abstain from eating, drinking, and smoking for one hour prior to arrival. Participants rinsed with water, then provided a saliva sample for baseline cortisol measurement via passive drool, which was immediately frozen.

All participants then received familiarization training on the Virtual Battle Space 2 (VBS2) desktop simulator and completed a practice scenario similar to the scenarios used in the pre- and post-training phases. Next, participants completed the pre-training phase of the experiment, which included a stress induction phase followed immediately by performance of the pre-training decision making under stress simulation scenario. The stress induction phase utilized either the Trier Social Stress Test (TSST; [36]), or the Socio-Evaluative Cold Press or Test (SECPT; [37]). The TSST is a well-established socio evaluative stressor consisting of 5 minutes each of: anticipatory stress; oral presentation; and mental arithmetic. The SECPT is a socio evaluative stressor that involves the participant immersing their full hand past their wrist in a bowl of ice water while being observed by a participant of the opposite sex [37]. Participants were then randomly assigned to either the BFT condition or the control condition and received training per their condition as described below. Following training, the participants completed the post-training phase of the experiment which included a stress induction phase followed immediately by performance of a different simulation-based decision making under stress scenario. All simulation scenarios

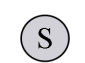
C

(c)

B

Familiarization
(S)

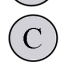

ECG, EDA

Figure 1. Experimental procedure. Following baseline physiological recording and familiarization with the simulator, participants were exposed to either the TSST or SECPT pre-training stressor. The pre-training simulation scenario followed immediately, followed by either BFT or control training. Following training, either the TSST or SECPT was presented as a post-training stressor, followed by post-training simulation scenario. B, baseline period; C, saliva sample for cortisol measurement; ECG, electrocardiogram measurement; EDA, electrodermal activity measurement; S, STAI administration. 
were approximately 15 - 20 minutes in length and included navigating through a virtual environment on a desktop PC utilizing a keyboard and mouse and monitoring auditory cues via a headset. Each scenario required the participant to respond to five stressful decision events with varying levels of unpredictability, uncontrollability, novelty, duration and intensity, five dimensions shown to impact stress response [38] [39]. In order to ensure each participant received a novel stressor during each stress induction phase, the stress induction techniques were counterbalanced with half of the participants in each treatment condition receiving the TSST in the pre-training phase and SECPT in the post-training and the other half of the participants in each treatment condition receiving the SECPT in the pre-training and the TSST in the post-training. Following the post-training phase, participants were debriefed and paid for their participation. The experiment lasted approximately four hours.

Physiological measures were captured throughout the baseline and stress induction phases. Cortisol and STAI measures were captured immediately following the baseline, pre-training and post-training phases. Performance was assessed throughout the simulation scenarios.

\subsection{Treatment Conditions}

Both treatment conditions followed a three-phase model similar to the BFT/SIT model discussed above, including: education (approx. 10 minutes), acquisition (approx. 15 minutes) and application (approx. 60 minutes). Total training time was approximately 85 minutes. For the BFT condition, the education phase consisted of a $10-15$ minute PowerPoint presentation focused on stress and its physiological impacts, the BFT method, and instructions on the diaphragmatic breathing technique which incorporated: 1) inhaling through the nose deeply, and expanding the stomach on a count of four, 2) holding that breath in for a count of four, 3) slowly exhaling through the mouth completely, and contracting the stomach for a count of four, and 4) holding that empty breath for a count of four [40]. During the acquisition phase, BFT participants recalled a series of particularly high-stress events (as used in [41]) while first monitoring the impact on their heart rate (an aural tone would sound when significantly higher than average baseline) and then attempting to lower their respiration and heart rate using the diaphragmatic breathing technique (as guided through the above four step process by a mobile application). During the application phase, BFT participants performed two decision making under stress simulation scenarios as described in [42] after being encouraged to incorporate the diaphragmatic breathing technique whenever they felt stressed and/or whenever they heard the aural tone indicating their heart rate was elevated. For the control condition, the education phase consisted of a 10 - 15 minute PowerPoint presentation focused on teaching the Simple Triage And Rapid Treatment (START) combat triage technique method and how to categorize patients into the different triage categories based on a series of cues. During the acquisition phase, the control condition participants were given a series of scenarios in which they practiced categorizing par- 
ticipants into the various triage categories. During the application phase, control participants performed two decision making under stress simulation scenarios.

\subsection{Measures}

Stress was assessed using real-time physiological measures of ANS response and cortisol measures of the HPA axis. Participants were fitted with a 3-lead electrocardiogram (ECG) with bandlimits set between 1 and $35 \mathrm{~Hz}$, and palmar electrodermal activity (EDA) on the $4^{\text {th }}$ and $5^{\text {th }}$ fingers of the non-dominant hand with bandlimits set between DC and $10 \mathrm{~Hz}$. All physiological data was sampled at $500 \mathrm{~Hz}$ and wirelessly sent to an MP-150 system running Acq Knowledge software (Biopac Systems, Goleta CA). Gain was set on ECG channels to 2000. The EDA data was run through digital bandpass filters at 1 and $0.05 \mathrm{~Hz}$, followed by thresholding between 0.05 and $0.051 \mu \mathrm{S}$ to identify electrodermal responses (EDR), which were quantified at a per minute rate. Heart rate was calculated from the R-R interval from the ECG, with intervals $<40 \mathrm{bpm}$ and $>180$ bpm excluded from the analysis. Briefly, baseline inter-beat interval (IBI) and heart rate (HR) data were used to normalize raw HR and IBI data throughout the study, in 1 minute non-overlapping windows, and a 2 -featuer linear model classifier was utilized to detect stress response. ANS stress response was quantified using methods described in [6]. Briefly, baseline HR and EDA were used to normalize real-time HR and EDA data. Normalized data was then subjected to a 2-feature linear model classifier which audibly alerted the participant when ANS stress was observed [43]. Salivary cortisol indicative of HPA stress response was measured by standard ELISA (Salimetrics, Carlsbad CA intra-assay CV $=4.5 \%$, inter-assay $\mathrm{CV}=5.8 \%$ ).

Perceived stress was measured at baseline and immediately following pre-training and post-training phases using the state portion of the STAI [34]. The STAI consists of 20 statements, and participants rank how closely the statement matches how they feel currently. Scores range from 20 to 80 , with higher scores indicating the presence of higher levels of stress.

Performance was measured within the simulation scenarios utilizing a researcher event-based evaluation checklist. For each of the five decision events in each scenario, observers captured participant decision making performance based on how well participants effectively observed critical cues, assessed the situation, selected an appropriate response and executed it. These scores were averaged across the five events of each scenario resulting in a scenario decision making performance score ranging from $0 \%-100 \%$.

\subsection{Data Analysis}

To account for individual differences, pre- and post-training cortisol and STAI measures were baseline normalized by dividing the difference between actual and baseline values by the baseline value. This resulted in percent change from baseline scores for cortisol and perceived stress. The data was then screened for missing physiological data and outliers; those participants who were missing a 
significant amount of physiological data or had ANS stress response data or cortisol data greater than two standard deviations from the mean during baseline, pre-training or post-training phases were excluded. The ANS stress response data that was outside of these thresholds were removed as it appeared to be due to sensor issues (e.g., loss of conductivity, broken leads, etc.). This resulted in inclusion of 31 participants, 17 within the BFT condition and 14 within the control condition. Socio demographic data associated with these 31 participants is summarized in Table 1 below.

Analysis of Variance (ANOVA) were performed with a within subjects factor of trial (pre-training vs. post-training) and a between subjects factor of condition (BFT vs. control) for measures of ANS stress response, cortisol, STAI and performance.

\section{Results}

\subsection{Autonomic Nervous System (ANS) Response}

Table 2 below shows the means and standard deviations of the unit-less, baseline normalized ANS stress response score for the BFT and control condition participants. The repeated measures ANOVA revealed that there was a significant trial effect for ANS stress response $\left(\mathrm{F}(1,29)=7.57, \mathrm{p}=0.01 ; \eta^{2}=0.21\right)$, with ANS stress response decreasing significantly for all participants from

Table 1. Socio demographic factors of study sample. Participants were an average age of $21.0 \pm 2.2$ years, and were generally currently pursuing a college education and had normal stress coping abilities as indicated by the Connors Davidson Resilience Scale (CDRISC).

Study sample \% (n)

$\begin{array}{cc}\text { Age Group } & \\ 18-20 & 38.7(12) \\ 21-23 & 45.2(14) \\ 24-26 & 16.1(5)\end{array}$

Education

High School Diploma

Some College/University

University Degree
$32.3(10)$

48.4 (15)

$19.4(6)$

Mean (SD)

CD-RISC $76.7(9.96)$

Table 2. ANS Stress response means and standard deviations.

\begin{tabular}{ccccc}
\hline \multirow{2}{*}{ Stress (No Unit) } & \multicolumn{2}{c}{ Biofeedback Training } & \multicolumn{2}{c}{ Control Condition } \\
\cline { 2 - 5 } & Mean & SD & Mean & SD \\
\hline Pre-training & 58.29 & 27.95 & 61.43 & 22.62 \\
Post-training & 43.59 & 33.14 & 51.71 & 20.02 \\
\hline
\end{tabular}




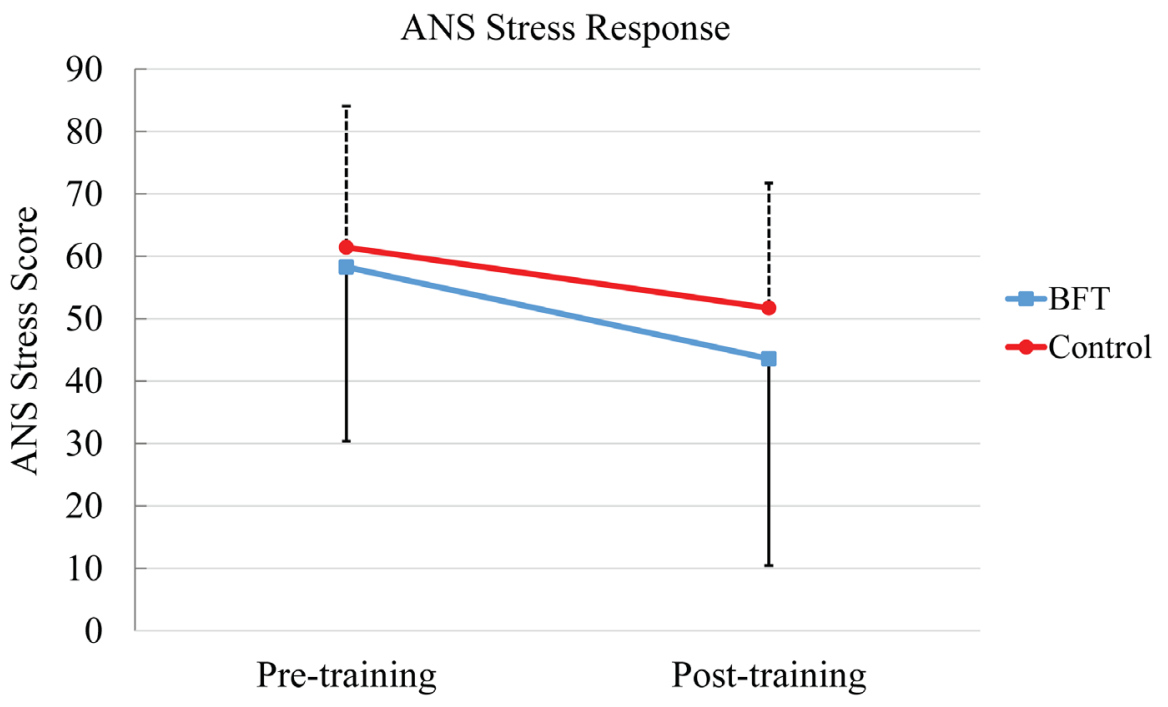

Figure 2. ANS stress response. Both the BFT and control groups significantly reduced ANS stress over the course of the experiment. No statistically significant group differences were observed.

Table 3. Cortisol percent change from baseline means and standard deviations.

\begin{tabular}{ccccc}
\hline \multirow{2}{*}{ Cortisol (\% Change) } & \multicolumn{2}{c}{ Biofeedback Training } & \multicolumn{2}{c}{ Control Condition } \\
\cline { 2 - 5 } & Mean & SD & Mean & SD \\
\hline Pre-training & 0.02 & 0.23 & 0.00 & 0.12 \\
Post-training & -0.04 & 0.21 & 0.12 & 0.29 \\
\hline
\end{tabular}

pre-training to post-training. There was not a significant group effect $(\mathrm{F}(1,29)=$ $\left.0.43, \mathrm{p}=0.52 ; \eta^{2}=0.01\right)$ or trial by group interaction $(\mathrm{F}(1,29)=0.32, \mathrm{p}=0.58$; $\eta^{2}=0.01$ ) for ANS stress response. See Figure 2.

\subsection{Cortisol Response (HPA Stress Response)}

Table 3 shows the means and standard deviations of the cortisol percent change from baseline for the BFT and control condition participants. The repeated measures ANOVA revealed that there was not a significant trial effect for cortisol response $\left(\mathrm{F}(1,29)=0.46, \mathrm{p}=0.50 ; \eta^{2}=0.02\right)$. There was also not a significant group effect $\left(\mathrm{F}(1,29)=1.11, \mathrm{p}=0.30 ; \eta^{2}=0.044\right)$ for cortisol. There was, however, a significant trial by group interaction $\left(\mathrm{F}(1,29)=5.16, \mathrm{p}=0.03 ; \eta^{2}=0.15\right)$ for cortisol response, with the BFT group having significantly greater reductions in cortisol response from pre-training to post-training compared to the control group which showed an increase from pre-training to post-training. See Figure 3.

\subsection{Perceived Stress}

Table 4 shows the means and standard deviations of the perceived stress percent change from baseline for the BFT and control condition participants as measured by the STAI. There was a significant trial effect for STAI scores $(F(1,29)=$ 4.23, $\left.\mathrm{p}=0.049 ; \eta^{2}=0.13\right)$, with STAI scores significantly increasing across 
Table 4. STAI percent change from baseline means and standard deviations.

\begin{tabular}{ccccc}
\hline \multirow{2}{*}{ STAI (\% Change) } & \multicolumn{2}{c}{ Biofeedback Training } & \multicolumn{2}{c}{ Control Condition } \\
\cline { 2 - 5 } & Mean & SD & Mean & SD \\
\hline Pre-training & 0.32 & 0.36 & 0.25 & 0.29 \\
Post-training & 0.37 & 0.38 & 0.47 & 0.58 \\
\hline
\end{tabular}

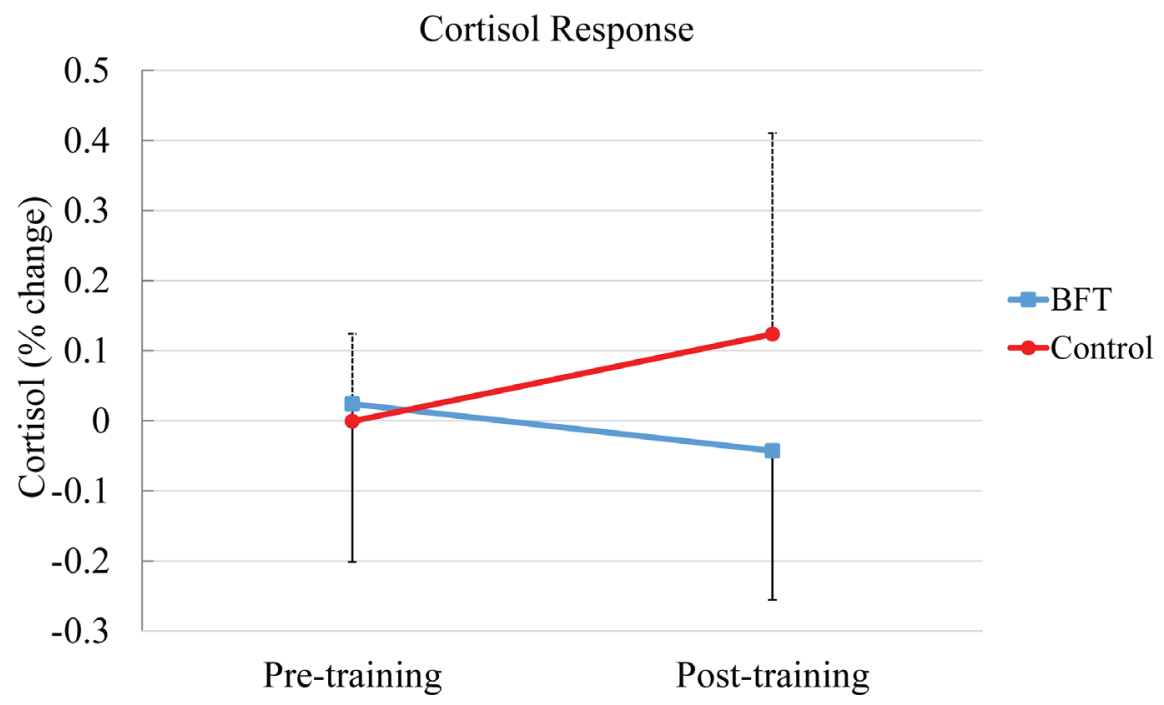

Figure 3. Cortisol response. The BFT group experienced a significant reduction in cortisol from pre-training to post-training, while the control group did not.

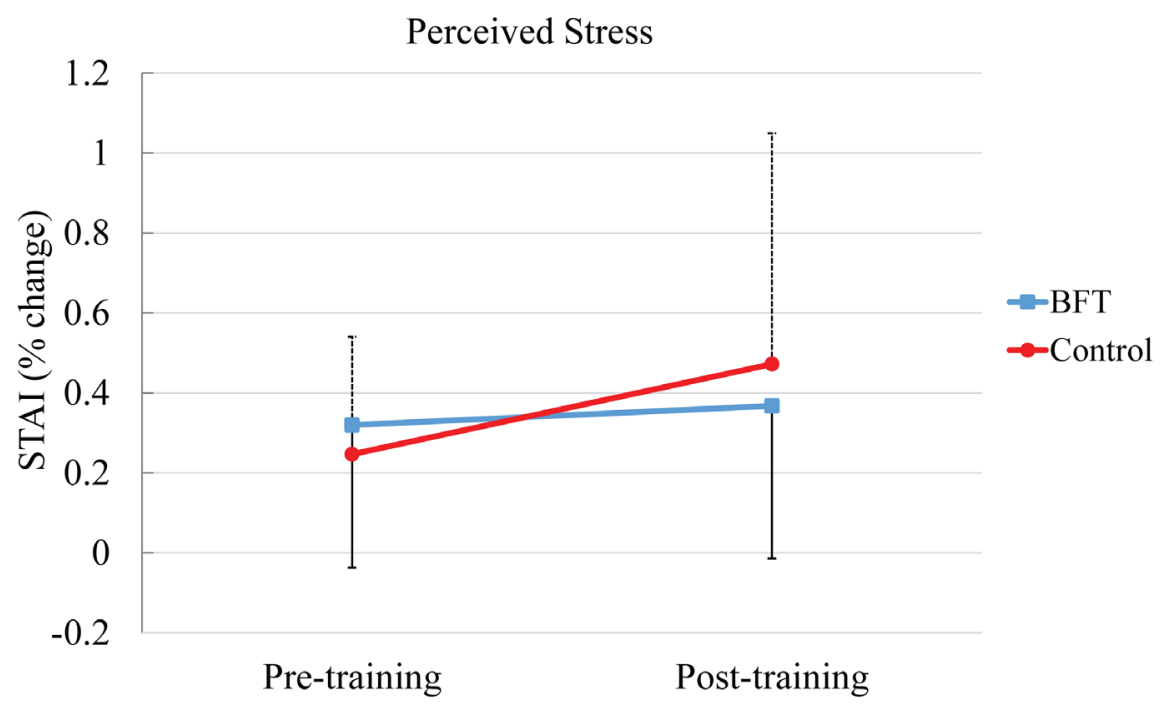

Figure 4. Perceived stress as measured by STAI percent change from baseline. There was a significant increase in STAI scores from pre-training to post-training across both groups.

all groups from pre-training to post-training. There was not a significant group effect $\left(\mathrm{F}(1,29)=0.012, \mathrm{p}=0.91 ; \eta^{2}=0.00\right)$ for STAI scores; nor was there a significant trial by group interaction $\left(\mathrm{F}(1,29)=1.81, \mathrm{p}=0.19 ; \eta^{2}=0.01\right)$. See Figure 4. 


\subsection{Performance}

Table 5 below shows the means and standard deviations of pre-training and post-training performance for the BFT and control condition participants. There was not a significant trial effect for performance $\left(\mathrm{F}(1,29)=1.44, \mathrm{p}=0.24 ; \eta^{2}=\right.$ $0.05)$. There was not a significant group effect $\left(\mathrm{F}(1,29)=0.25, \mathrm{p}=0.62 ; \eta^{2}=\right.$ $0.01)$ for performance, nor was there a significant trial by group interaction $(\mathrm{F}(1$, 29) $=3.01, \mathrm{p}=0.09 ; \eta^{2}=0.09$ ). See Figure 5 below.

\section{Discussion}

The objective of this study was to examine the effectiveness of a condensed BFT method at reducing acute stress responses and improving performance under stress. It was hypothesized that the BFT method would lead to significant reductions in physiological stress responses as indicated by measures of both the ANS (aggregate measure of HR and EDA) and the HPA Axis (cortisol). This hypothesis was partially supported. Participants in the BFT condition did experience a significant reduction in cortisol from pre-training to post-training, while the control group did not. In fact, BFT participants experienced a $4 \%$ decrease in cortisol response from baseline compared to the control group who actually experienced a $12 \%$ increase. This is in-line with findings from [17] who found BFT led to a decrease in cortisol response compared to an increase in control condition cortisol response. These levels are also in-line with cortisol reduction levels

Table 5. Performance means and standard deviations.

\begin{tabular}{ccccc}
\hline \multirow{2}{*}{ Performance (\%) } & \multicolumn{2}{c}{ Biofeedback Training } & \multicolumn{2}{c}{ Control Condition } \\
\cline { 2 - 5 } & Mean & SD & Mean & SD \\
\hline Pre-training & 49 & 22 & 59 & 16 \\
Post-training & 62 & 19 & 57 & 22 \\
\hline
\end{tabular}

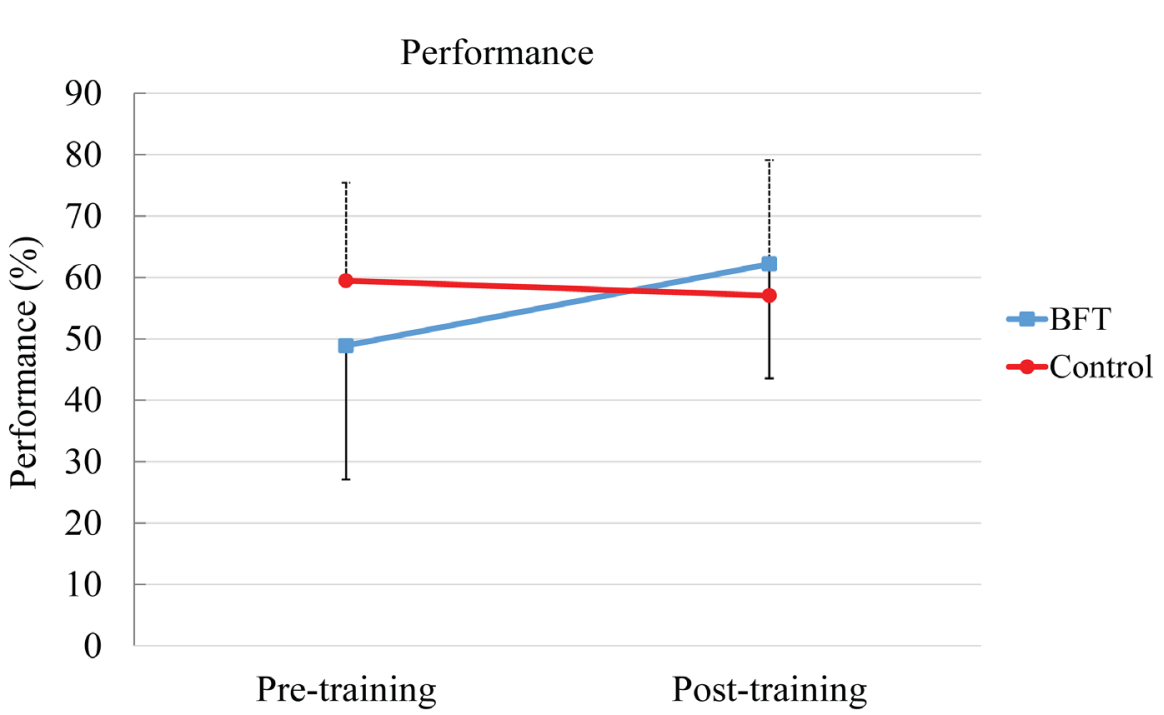

Figure 5. Performance scores. BFT group experienced greater improvements in performance from pre-training to post-training compared to the control group, however, these results only approached statistical significance $(\mathrm{p}=0.09)$. 
observed in [24] who observed an approximate 7\% decrease in cortisol from baseline after BFT. These findings suggest BFT participants were able to implement strategies which led to reduction of HPA stress response. However, BFT participants did not experience significantly greater reductions in ANS stress response compared to control participants. Participants across both groups exhibited significant decreases in ANS stress response from pre-training to posttraining with BFT participants experiencing a 25\% decrease in ANS stress response compared to the control group who experienced a $16 \%$ decrease. This is not in-line with findings associated with longer BFT programs (e.g., [31]) or condensed BFT programs [18] both of which found significantly greater shifts in cardiac measures such as HRV when compared to a control group. While such measures did not reach statistical significance, they may be functionally significant. Further, there are several reasons why this difference may not have reached statistical significance. The physiological measures utilized to assess ANS activity are proxy measures of underlying neural systems and may not reflect precise sympathetic activation. In addition, a reduction in physiological metrics associated with stress was observed in both the control and experimental group for the second stress induction phase, which may be associated with reduced novelty to the laboratory environment and experimenters over time [39] [44]. The magnitude of this effect may have served to effectively dilute the BFT training effect. Alternatively, the reduction in stress metrics during the course of the experiment may be due to natural circadian decay across the duration of the study [45]. However, since there was no observation of a decrease in salivary cortisol in the control group, we infer the cortisol decrease in the BFT group to be due to the shortened BFT training. Previous research has indicated that the results of a short term stress intervention on HPA and ANS stress responses may not manifest immediately, and may take a significant amount of time (several weeks) to be manifested [46].

An additional hypothesis of the study was that the shortened BFT would lead to significant reductions in perceived stress and significant increases in performance under stress. Participants in the BFT condition did not experience significantly greater reductions in perceived stress compared to the control group. On the contrary, participants across both groups experienced a significant increase in perceived stress as measured by the STAI, however, the BFT condition experienced only a moderate increase in percent change from baseline (5\%) while the control participants experienced a much greater increase in percent change from baseline (22\%). These are not unlike findings from [32] that reported no change in perceived stress measured via the STAI after BFT, despite BFT resulting in significant decreases in cortisol up to 8 months after the training. Further, observations between objective and subjective stress markers are frequently not in agreement and may be associated with observation or response bias [47]. Participants in the biofeedback group also experienced greater improvements in performance from pre-training to post-training compared to the control group, however, these results only approached statistical significance $(p=0.09)$. The 
BFT group showed a $13 \%$ increase from pre-training to post-training compared to the control group who experienced a $2 \%$ decrease. These results suggest the effectiveness of the stress coping strategies learned and implemented by the BFT group (as evident by the reduction in cortisol levels) may have led to improved decision making performance. This is consistent with decision making under stress research which has shown stress to have a range of negative impacts on decision making performance including attentional narrowing and reduction in environmental scan, perseveration, and reduction in the number and quality of alternatives considered [48] [49]. Lack of statistically significant performance improvements might be due to potential increases in cognitive load associated with monitoring of physiological indicators and implementation of the diaphragmatic breathing technique.

\section{Limitations}

Multiple limitations may have impacted the outcome of the study. Two different stressors were utilized in the experiment, since an individual stress induction technique will present decreased stressfulness if repeated [39]. To date, there has been no quantitative comparison of the TSST and SECPT, but previous research has indicated that both stressors have a large impact on cortisol reactivity [36] [37]. While the stressors were counterbalanced, there are significant differences in the stressor duration and type that may impact the experimental findings. In addition, the stress associated with the scenarios was likely perceived to be quite low, as has been observed in previous video game-based stress reports [39] [44]. An additional limitation was the quality of the physiological sensor data. Due to the length of the study and the placement of EDA sensors on the hands while asking participants to use their hands to interact with a computer, a number of participants experienced significant movement artifact and loss of signal.

\section{Conclusion}

Taken together, these results suggest that the condensed BFT method has the potential to impart the knowledge and skills necessary to implement the biofeedback-based coping mechanisms; however, it may require additional practice time to allow the technique to be utilized more effectively and automatically. Future research should also examine the cognitive load associated with implementation of the BFT technique. Additionally, future research should attempt to accomplish such a study over a shorter period of time in order to increase the accuracy and reliability of ANS physiological measures and decrease the impact of natural circadian decay throughout the day. Future research should also examine the longitudinal impact of BFT-based stress reduction on the development of clinical stress disorders, disease states associated with stress, and resilience in the face of new stressors.

\section{Acknowledgements}

This research was sponsored by the Office of Naval Research under contract 
N00014-12-G-0427. Publication of this article was funded in part by the Open Access Subvention Fund and the Florida Tech Libraries.

\section{References}

[1] Lieberman, H.R., et al. (2005) Severe Decrements in Cognition Function and Mood Induced by Sleep Loss, Heat, Dehydration, and Undernutrition during Simulated Combat. Biological Psychiatry, 57, 422-429.

https://doi.org/10.1016/j.biopsych.2004.11.014

[2] McHugh, R.K., et al. (2010) Cortisol, Stress, and Attentional Bias toward Threat. Anxiety Stress Coping, 23, 529-545. https://doi.org/10.1080/10615801003596969

[3] van Wingen, G.A., et al. (2012) Persistent and Reversible Consequences of Combat Stress on the Mesofrontal Circuit and Cognition. Proceedings of the National Academy of Sciences of the United States of America, 109, 15508-15513. https://doi.org/10.1073/pnas.1206330109

[4] Cohen, S., Janicki-Deverts, D. and Miller, G.E. (2007) Psychological Stress and Disease. JAMA, 298, 1685-1687. https://doi.org/10.1001/jama.298.14.1685

[5] Cooper, C.L. (1995) Identifying Workplace Stress: Costs, Benefits, and the Way Forward. New Solutions. A Journal of Environmental and Occupational Health Policy, 4, 38-40. https://doi.org/10.2190/NS4.4.g

[6] Pitman, R.K., et al. (2012) Biological Studies of Post-Traumatic Stress Disorder. Nature Reviews Neuroscience, 13, 769-787. https://doi.org/10.1038/nrn3339

[7] Morgan, C.A., Doran, A., Steffian, G., Hazlett, G. and Southwick, S.M. (2006) Stress-Induced Deficits in Working Memory and Visuo-Constructive Abilities in Special Operations Soldiers. Biological Psychiatry, 60, 722-729.

https://doi.org/10.1016/j.biopsych.2006.04.021

[8] Baker, D.G., Nash, W.P., Litz, B.T., Geyer, M.A., Risbrough, V.B., Nievergelt, C.M., et al. (2012) Predictors of Risk and Resilience for Posttraumatic Stress Disorder among Ground Combat Marines: Methods of the Marine Resiliency Study. Preventing Chronic Disease, 9, E97. https://doi.org/10.5888/pcd9.110134

[9] Milliken, C.S., Auchterlonie, J.L. and Hoge, C.W. (2007) Longitudinal Assessment of Mental Health Problems among Active and Reserve Component Soldiers Returning from the Iraq War. JAMA, 298, 2141-2148. https://doi.org/10.1001/jama.298.18.2141

[10] Rosenkranz, M.A., et al. (2013) A Comparison of Mindfulness-Based Stress Reduction and an Active Control in Modulation of Neurogenic Inflammation. Brain, Behavior, and Immunity, 27, 174-184.

[11] Wadlinger, H.A. and Isaacowitz, D.M. (2011) Fixing Our Focus: Training Attention to Regulate Emotion. Personality and Social Psychology Review, 15, 75-102. https://doi.org/10.1177/1088868310365565

[12] Jha, A.P., Stanley, E.A., Kiyonaga, A., Wong, L. and Gelfand, L. (2010) Examining the Protective Effects of Mindfulness Training on Working Memory Capacity and Affective Experience. Emotion, 10, 54-64. https://doi.org/10.1037/a0018438

[13] Butler, A.C., Chapman, J., Forman, E. and Beck, A. (2006) The Empirical Status of Cognitive-Behavioral Therapy: A Review of Meta-Analyses. Clinical Psychology Review, 26, 17-31.

[14] Schnurr, P.P., et al. (2007) Cognitive Behavioral Therapy for Posttraumatic Stress Disorder in Women: A Randomized Controlled Trial. JAMA, 297, 820-830. https://doi.org/10.1001/jama.297.8.820

[15] Calderon, K.S. and Thompson, W.W. (2004) Biofeedback Relaxation Training: A 
Rediscovered Mind-Body Tool in Public Health. American Journal of Health Studies, 19, 185.

[16] Frank, D.L., et al. (2010) Biofeedback in Medicine: Who, When, Why and How? Mental Health in Family Medicine, 7, 85-91.

[17] Kotozaki, Y., et al. (2014) Biofeedback-Based Training for Stress Management in Daily Hassles: An Intervention Study. Brain and Behavior, 4, 566-579. https://doi.org/10.1002/brb3.241

[18] Lewis, G.F., et al. (2015) Relaxation Training Assisted by Heart Rate Variability Biofeedback: Implication for a Military Predeployment Stress Inoculation Protocol. Psychophysiology, 52, 1167-1174. https://doi.org/10.1111/psyp.12455

[19] Strack, B.W. (2003) Effect of Heart Rate Variability (HRV) Biofeedback on Batting Performance in Baseball. ProQuest Information \& Learning.

[20] Ford, K.R., DiCesare, C.A., Myer, G.D. and Hewett, T.E. (2015) Real-Time Biofeedback to Target Risk of Anterior Cruciate Ligament Injury: A Technical Report for Injury Prevention and Rehabilitation. Journal of Sport Rehabilitation, Technical Notes 13.

[21] Ratanasiripong, P., Sverduk, K., Hayashino, D. and Prince, J. (2010) Setting up the Next Generation Biofeedback Program for Stress and Anxiety Management for College Students: A Simple and Cost-Effective Approach. College Student Journal, 44, 97-100.

[22] Saunders, T., Driskell, J.E., Hall, J. and Salas, E. (1996) The Effect of Stress Inoculation Training on Anxiety and Performance. Educational Publishing Foundation.

[23] Driskell, J.E. and Johnston, J.H. (1998) Stress Exposure Training. In: CannonBowers, J.A. and Salas, E., Eds., Making Decisions under Stress. Implications for Individual and Team Training, American Psychological Association, Washington DC, 191-217. https://doi.org/10.1037/10278-007

[24] Bouchard, S., Bernier, F., Boivin, É., Morin, B. and Robillard, G. (2012) Using Biofeedback While Immersed in a Stressful Videogame Increases the Effectiveness of Stress Management Skills in Soldiers. PLoS ONE, 7, e36169. https://doi.org/10.1371/journal.pone.0036169

[25] Gaab, J., Blättler, N., Menzi, T., Pabst, B., Stoyer, S. and Ehlert, U. (2003) Randomized Controlled Evaluation of the Effects of Cognitive-Behavioral Stress Management on Cortisol Responses to Acute Stress in Healthy Subjects. Psychoneuroendocrinology, 28, 767-779. https://doi.org/10.1016/S0306-4530(02)00069-0

[26] Meichenbaum, D. (1985) Stress Inoculation Training. Pergamon Press, New York.

[27] Sheehy, R. and Horan, J.J. (2004) Effects of Stress Inoculation Training for 1st-Year Law Students. International Journal of Stress Management, 11, 41-55. https://doi.org/10.1037/1072-5245.11.1.41

[28] Hourani, L.L., et al. (2011) Predeployment Stress Inoculation Training for Primary Prevention of Combat-Related Stress Disorders. Journal of CyberTherapy and Rehabilitation, 4, 101-117.

[29] Reiner, R. (2008) Integrating a Portable Biofeedback Device into Clinical Practice for Patients with Anxiety Disorders: Results of a Pilot Study. Applied Psychophysiology and Biofeedback, 33, 55-61. https://doi.org/10.1007/s10484-007-9046-6

[30] Riegel, B., et al. (1995) Psychogenic Cough Treated with Biofeedback and Psychotherapy: A Review and Case Report. American Journal of Physical Medicine \& Rehabilitation, 74, 155-158.

[31] Purwandini Sutarto, A., Wahab, M.N.A. and Zin, N.M. (2012) Resonant Breathing Biofeedback Training for Stress Reduction among Manufacturing Operators. Inter- 
national Journal of Occupational Safety and Ergonomics, 18, 549-561. https://doi.org/10.1080/10803548.2012.11076959

[32] Davis, H. (1986) Effects of Biofeedback and Cognitive Therapy on Stress in Patients with Breast Cancer. Psychological Reports, 59, 967-974.

https://doi.org/10.2466/pr0.1986.59.2.967

[33] Cook-Vienot, R. and Taylor, R.J. (2012) Comparison of Eye Movement Desensitization and Reprocessing and Biofeedback/Stress Inoculation Training in Treating Test Anxiety. Journal of EMDR Practice and Research, 6, 62-72. https://doi.org/10.1891/1933-3196.6.2.62

[34] Spielberger, C.D., Gorsuch, R.L., Lushene, R.E. and Vagg, P.R. (1983) State-Trait Anxiety Inventory (STAI). BiB, 2010, 180.

[35] Rule, W.R. and Traver, M.D. (1983) Test-Retest Reliabilities of State-Trait Anxiety Inventory in a Stressful Social Analogue Situation. Journal of Personality Assessment, 47, 276-277. https://doi.org/10.1207/s15327752jpa4703_8

[36] Kirschbaum, C., Pirke, K.-M. and Hellhammer, D.H. (1993) The "Trier Social Stress Test"-A Tool for Investigating Psychobiological Stress Responses in a Laboratory Setting. Neuropsychobiology, 28, 76-81. https://doi.org/10.1159/000119004

[37] Schwabe, L., Haddad, L. and Schachinger, H. (2008) HPA Axis Activation by a Socially Evaluated Cold-Pressor Test. Psychoneuroendocrinology, 33, 890-895. https://doi.org/10.1016/j.psyneuen.2008.03.001

[38] Bouchard, S., Baus, O., Bernier, F. and McCreary, D.R. (2010) Selection of Key Stressors to Develop Virtual Environments for Practicing Stress Management Skills with Military Personnel Prior to Deployment. Cyberpsychology, Behavior, and Social Networking, 13, 83-94. https://doi.org/10.1089/cyber.2009.0336

[39] Dickerson, S.S. and Kemeny, M.E. (2004) Acute Stressors and Cortisol Responses: A Theoretical Integration and Synthesis of Laboratory Research. Psychological Bulletin, 130, 355-391. https://doi.org/10.1037/0033-2909.130.3.355

[40] Grossman, D. and Christensen, L.W. (2007) On Combat: The Psychology and Physiology of Deadly Conflict in War and in Peace. PPCT Research Publications, Belleville, IL.

[41] Kimbrell, T.A., et al. (1999) Regional Brain Activity during Transient Self-Induced Anxiety and Anger in Healthy Adults. Biological Psychiatry, 46, 454-465. https://doi.org/10.1016/S0006-3223(99)00103-1

[42] Winslow, B., Carroll, M.B., Martin, J.W., Surpris, G. and Chadderdon, G.L. (2015) Identification of Resilient Individuals and Those at Risk for Performance Deficits under Stress. Frontiers in Neuroscience, 9, 328. https://doi.org/10.3389/fnins.2015.00328

[43] Winslow, B.D., et al. (2016) Development and Clinical Evaluation of an mHealth Application for Stress Management. Frontiers in Psychiatry, 7, 130.

[44] Biondi, M. and Picardi, A. (1999) Psychological Stress and Neuroendocrine Function in Humans: The Last Two Decades of Research. Psychotherapy and Psychosomatics, 68, 114-150. https://doi.org/10.1159/000012323

[45] Weibel, L., Spiegel, K., Follenius, M., Ehrhart, J. and Brandenberger, G. (1996) Internal Dissociation of the Circadian Markers of the Cortisol Rhythm in Night Workers. American Journal of Physiology, 270, E608-E613.

[46] De Brouwer, S.J., et al. (2011) Psychophysiological Responses to Stress after Stress Management Training in Patients with Rheumatoid Arthritis. PLoS ONE, 6, e27432. https://doi.org/10.1371/journal.pone.0027432

[47] Michaud, D.S., Feder, K., Keith, S.E. and Voicescu, S.A. (2016) Self-Reported and 
Measured Stress Related Responses Associated with Exposure to Wind Turbine Noise. The Journal of the Acoustical Society of America, 139, 1467.

https://doi.org/10.1121/1.4942402

[48] Staal, M.A. (2004) Stress, Cognition, and Human Performance: A Literature Review and Conceptual Framework.

[49] Woods, D.D., et al. (1994) Behind Human Error: Cognitive Systems, Computers and Hindsight. DTIC Document.

Submit or recommend next manuscript to SCIRP and we will provide best service for you:

Accepting pre-submission inquiries through Email, Facebook, LinkedIn, Twitter, etc. A wide selection of journals (inclusive of 9 subjects, more than 200 journals)

Providing 24-hour high-quality service

User-friendly online submission system

Fair and swift peer-review system

Efficient typesetting and proofreading procedure

Display of the result of downloads and visits, as well as the number of cited articles

Maximum dissemination of your research work

Submit your manuscript at: http://papersubmission.scirp.org/

Orcontact jbbs@scirp.org 